\title{
Omics Conferences and Breast Cancer in Sub-Saharan Africa
}

\section{Retsky $\mathbf{M}^{*}$}

Editor in Chief Journal of Bioequivalence and Bioavailability, Harvard TH Chan School of Public Health, USA

In a previous editorial, I discussed a cancer research project underway and how this may lead to more use of bioequivalence and bioavailability in oncology [1]. Recently there has been a development and it relates to Omics conferences and breast cancer.

Permit me an observation. With the advent of open access publishing, there seems to be less need to attend distant conferences where the typical attendees are high level people who are thought leaders in specialized fields. These people frequently publish papers and, using modern open access, anyone can download and read their papers (Omics has played an important role in promoting open access). Why travel to meetings where your peers discuss their published work in front of others interested in the same field? You can more conveniently and with less stress to your budget read their papers in the comfort of your office or home lounge chair.

In comparison, I have attended over 10 Omics conferences. The thing I like best about Omics conferences and why I keep going there is the opportunity to meet people from other countries and cultures who may discuss community based research and might have something of interest to say or provide a new perspective or research opportunity. As one notable recent example, I was attending an Omics conference in Baltimore, MD last October accompanied by my wife. There were 4 or 5 separate Omics conferences going on concurrently in the same hotel and there were many people I did not know. At a lunch break my wife and I decided to sit at a table where there were 7 people already present who all looked to be Africans. They were the best dressed persons at the conference and were attending a hematology-oncology meeting. I did not know any of them but my wife who has extremely good social skills made sure everyone introduced themselves and got the conversation going among us.

Eventually the conversation got around to the breast cancer research I am doing with my colleagues from Italy, Belgium, and the UK. I discussed that based on a retrospective study this research might result in a low cost and low toxicity intervention that could dramatically reduce relapses from early to late stage disease [2]. By low cost, I mean a $\$ 1.92$ drug given once. The problem we have encountered is that paradoxically the therapy is too inexpensive. That is, it needs to be confirmed in a randomized controlled clinical trial that can cost significant funds to conduct but there is no financial reason to justify such a trial by a conventional pharmaceutical company. It is not that these people are evil. It is just a financial reality. If I were working for a pharmaceutical company instead of doing academic research I would probably not request management to spend millions of dollars to support a project that will show no financial return even if scientifically successful. To add to our inability to get financial support, as a policy rule the US NIH does not support phase III trials which is what is needed. We submitted three grant proposals to support confirming clinical trials in India and all were rejected. This may be an inexpensive solution to an expensive problem. Absurdity does not begin to describe this bizarre situation but it is real [3]. According to clinicaltrials.gov there is a small trial underway in Brussels, Belgium and another is planned in Seoul, South Korea so there may be results to show in upcoming years.

As an unexpected result of my chance encounter at the Baltimore
Omics conference, I have been invited to a conference in Nigeria. The underlying science is that our therapy - perioperative use of a common analgesic NSAID in breast cancer surgery - might reduce relapse and eventual mortality in breast and other cancers. It is relevant that the Nigerian population might be the best possible group to show benefit from this therapy. This proposed benefit would appear within 1-3 years after primary surgery. The therapy should work best for triple negative breast cancer in which case there is no effective targeted therapy indicated. Only adjuvant chemotherapy has shown some value. Triple negative is not very common in the US but is more frequently seen in other biologically distinct groups especially Koreans, Indians and SubSaharan Africans. A key indicator is the age at diagnosis. Typically the type of breast cancer we think should respond best is for women who present at an early age - say in their 40 s compared to 50 s as is most often the case in the US [4]. With a large percentage of triple negative breast cancer in the Nigerian population, we could determine if there is the expected advantage with relatively few patients and in a relatively short time.

This connection is totally the result of unplanned networking at the Baltimore 2014 Omics conference. I recommend that you attend Omics conferences, acquire social skills, and make an effort to meet and speak to people from other parts of the world. I will report more after the conference in Nigeria that is scheduled for July 8-12, 2015 in Obaju and in Sokoto on July 13.

\section{Conflicts of Interest}

Michael Retsky is on the board of directors of the Colon Cancer Alliance, is Chief Scientific Officer at GR Enterprises and Technologies, Inc. and has a patent pending for treatment of early stage cancer. No other conflicts to report.

\section{References}

1. Retsky M (2015) BABE is A Beautiful Science: Why is it Not More Often Used in Oncology? J Bioequiv Availab 7:e63.

2. Retsky M, Demicheli R, Hrushesky WJ, Forget P, De Kock M, et al. (2013) Reduction of breast cancer relapses with perioperative non-steroidal antiinflammatory drugs: new findings and a review. Curr Med Chem 20: 41634176.

3. http://www.propublica.org/article/where-are-the-low-cost-cancer-treatments

4. Demicheli R, Retsky MW, Hrushesky WJ, Baum M, Gukas ID, et al. (2007) Racial disparities in breast cancer outcome: insights into host-tumor interactions. Cancer 110: 1880-1888.

*Corresponding author: Michael Retsky, Harvard T.H. Chan School of Public Health, Boston, MA 02115, USA and University College London, UK, Tel: 203-6750017; E-mail: michael.retsky@gmail.com

Received May 14, 2015; Accepted May 15, 2015; Published May 22, 2015

Citation: Retsky M (2015) Omics Conferences and Breast Cancer in Sub-Saharan Africa. J Bioequiv Availab 7: e66. doi:10.4172/jbb.10000e66

Copyright: (c) 2015 Retsky M. This is an open-access article distributed under the terms of the Creative Commons Attribution License, which permits unrestricted use, distribution, and reproduction in any medium, provided the original author and source are credited. 